\title{
VALORES PARA EL TRABAJO: SUS POSIBILIDADES DE APLICACIÓN A LA FORMACIÓN EN EL NUEVO CONTEXTO SOCIOECONÓMICO DE LA GLOBALIZACIÓN
}

\author{
Chantal Biencinto López \\ Juan Antonio García Fraile \\ Universidad Complutense
}

\begin{abstract}
RESUMEN: El presente artículo tiene como objetivo la creación de un escenario conceptual que nos sirva de referencia cuando hablamos de valores en o para el trabajo. Este recorrido teórico está inmerso en la sociedad del conocimiento y la globalización, marco donde podemos situar a la educación en general y a la formación en particular. Nos referiremos a la aplicación de estos valores en distintos sectores productivos y por tanto en distintos ámbitos formativos.
\end{abstract}

ABSTRACT: The present article has like objective the creation of a conceptual scene that serves to us as reference when we spoke of values in or for the work. This theoretical route this immersed in the society of the knowledge and the globalization, frame where we can locate to the education in general and the training in individual. We will talk about to the application of these values in different productive sectors and therefore in different traine scopes.

PALABRAS CLAVE: Valores para el trabajo, formación, globalización.

KEY WORDS: Values, training, globalization.

\section{INTRODUCCIÓN}

La educación es una condición previa para el empleo y quizá el activo más importante de una persona al enfrentarse a un porvenir incierto. Sin embargo, no es una salvaguarda frente a un posible desempleo futuro, aunque normalmente mejore las posibilidades de encontrar trabajo. Una buena formación prepara al individuo para afrontar las demandas de la sociedad futura y de su sistema económico.

Como consecuencia de lo anterior, durante la década de los noventa, comenzó a manifestarse una preocupación mundial por el comportamiento del ser 
humano y sus consecuencias en todos los ámbitos. Algunas de las causas señaladas son la pérdida de la conciencia social, el descuido de la educación de las nuevas generaciones, (entendida como la formación total del ser humano, no sólo los niveles académicos que se pueden alcanzar), y la pérdida de los valores y el respeto social e individual.

A partir de ello comenzaron a surgir una serie de corrientes en el pensamiento social que conllevaban una búsqueda de soluciones a este fenómeno. Así, apareció el Paradigma "Desarrollo Sostenible" y con él, la vuelta al "neohumanismo", donde destacan elementos tales como el rescate de las normas morales y la ética, la Libertad, la Democracia, y los Derechos Humanos, recordando que la responsabilidad de los cambios "positivos y negativos" que tiene el entorno, social y natural, son competencia de la propia sociedad en la que él convive.

La sociedad, no ha sido ajena a este proceso, sino que se ha visto involucrada totalmente en él, ya que uno de los objetivos de este nuevo paradigma es generar un cambio positivo en el comportamiento de los grupos humanos. Por esto, se debe aceptar que parte de la función como miembros de un grupo social es la transmisión de estas corrientes a las nuevas generaciones.

En todos los casos hay que asegurarse de que la calidad de la educación y la formación se correspondan lo más posible con las futuras necesidades. No es fácil imaginar mayor pérdida de esfuerzo e idealismo que en el caso de una población altamente cualificada que satisface el nivel general de calidad del mercado laboral pero no sus demandas, por no contar con las aptitudes y valores adecuados (Giarini y Liedtke, 1998: 65).

\section{LOS VALORES SOCIALES E INDIVIDUALES: ÉTICA Y MORAL}

La filosofía maneja esta vertiente del pensamiento humano, a través de la Axiología, definida como la parte de la Filosofía que estudia los valores, especialmente morales. Esta, divide a los Valores en sociales, denominados "ética"; e individuales, englobados en la "moral". En ambos casos los valores principales se aplican de manera directa, pero además, se debe aceptar que si un valor no se aplica en lo individual, no se ejercerá hacia los demás miembros de la sociedad.

La moral es un elemento que se asocia a las creencias religiosas, y en muchos casos a esta parte de la educación "espiritual" es a la que se deja como responsabilidad de otros. La ética, por otro lado, es algo que muchos creen sólo se debe aplicar en cuanto a la profesión que se desempeña, pero la filosofía marca que el comportamiento "ético" debe darse en cualquier actividad que se desempeñe.

Lo anterior pone de manifiesto a que valor o conjunto de valores le da más importancia cada cultura. Dentro de este marco, los valores se han clasificado de la siguiente manera (tabla 1): 
Tabla 1

\begin{tabular}{|c|c|c|c|c|c|}
\hline Valores vitales & \multicolumn{2}{|c|}{ Capacidad física } & Salud & \multicolumn{2}{|c|}{ Vigor } \\
\hline Valores hedónicos & Placer $\quad \mathrm{Fe}$ & Felicidad & Alegría & Solaz & \\
\hline Valores económicos & \multicolumn{2}{|c|}{ Valor de uso } & \multicolumn{2}{|c|}{ Valor de cambio } & \\
\hline Valores estéticos & Belleza & Elegancia & \multicolumn{2}{|c|}{ Gracia } & \\
\hline Valores religiosos & Santidad & \multicolumn{2}{|c|}{ Bienaventuranza } & \multicolumn{2}{|l|}{ Piedad } \\
\hline Valores de la ciencia & Probabilidad & \multicolumn{2}{|c|}{ Exactitud } & Verdad & \\
\hline Valores individuales & Templanza & Valentía & Bondad & Justicia & Veracidad \\
\hline
\end{tabular}

Todo lo anterior nos lleva a ver que existen dos tipos de valores, aplicados los diferentes entornos de convivencia (tabla 2).

Tabla 2

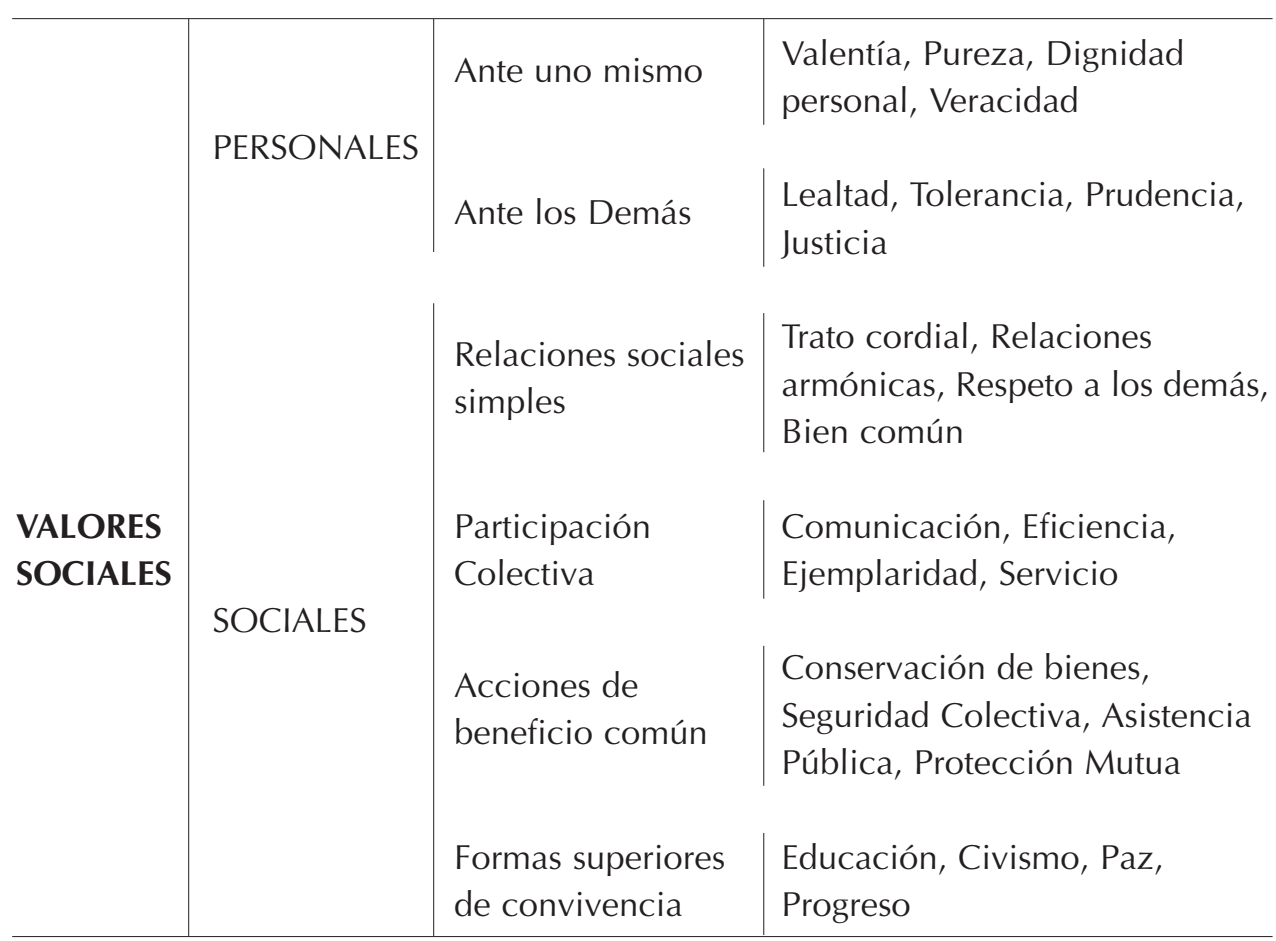

Existen muchas más percepciones, pero la mayoría de los autores coinciden en que los valores tienen dos características básicas: su origen humano, y su naturaleza abstracta. Lo primero, se percibe en la herencia cultural de cada sociedad, pues es donde se encuentran insertados; lo segundo, manifiesta su existencia ideal. Se debe partir de cuatro puntos básicos para estudiar los valores: 
$\checkmark$ IDEAL: por ser una creación humana, es decir, es un bien cultural, su campo de aparición es la esfera de lo abstracto donde los valores nacen. Desde este ángulo los valores son parámetros de comportamiento que una sociedad acepta y se mantienen como lo que se debe alcanzar, la meta a lograr. Estos reflejan los elementos estéticos, comportamiento aceptable, relaciones humanas, la moral y la ética, entre otros.

$\checkmark$ EMPÍRICO: es donde el hombre aplica los valores. Los valores que trasmite cada sociedad a sus miembros de manera directa con el comportamiento, por lo que son aceptados como algo "natural", y no como algo "aprendido" y "razonado". En algunos casos son incuestionables.

$\checkmark$ PERSONAL: el ser humano es quien le atribuye un valor a los elementos de su entorno, siempre y cuando el objeto le signifique algo, por su esencia y lo que representa en sí mismo, como cualidad de un bien cultural. Por ejemplo, el trabajo tiene un valor por el producto obtenido, y otro por la satisfacción de haberlo hecho bien; o un objeto tiene un valor por su forma y otro por lo que significa (recuerdo, regalo, cariño).

$\checkmark$ CULTURAL: en todos los grupos sociales los valores son el ideal a seguir, el máximo estadio del espíritu humano, es decir, es la aspiración a buscar lo mejor, lo más alto; como respetar la naturaleza para tener un mejor entorno; ser honesto en la profesión, ser responsable como padre, hijo y hermano; entre otros.

\section{Características de los Valores}

Los valores son criterios de comportamiento y motivos de conducta y sus características fundamentales son:

- Polaridad: los valores siempre se manejan en dos extremos, uno positivo y otro negativo (valor, contravalor); según el grado de indiferencia del sujeto, su atracción o aversión.

- Gradación: intensidad o abundancia de generación y reconocimiento de valores, positivos o negativos, conforme al ideal determinado entre los extremos.

- Cualidad: clasificación de los valores en superior e inferior, medida más cualitativa que cuantitativa

- Preferibilidad: el valor que se elige y se prefiere en anteposición de una cosa a otra. Los valores son un DEBER SER, en contraposición a los hechos, o a las cosas existentes: hay cosas que encarnan los valores, no hay valores que sean cosas; lo que caracteriza al valor no es el ser, sino él deber ser.

- Idealidad: son bienes culturales procedentes del espíritu y sólo la mente puede percibirlos como objetos.

- Modalidad: cada valor se relaciona con un elemento cultural, cada uno soporta su propio valor y cada valor se refleja en el bien que lo contiene. Por ejemplo, los valores artísticos se aprecian en el arte, los valores éticos en el comportamiento individual, y los valores docentes en la educación. 
- Jerarquización: los valores tienen un orden escalonado de acuerdo al grado poseído para quitar la indiferencia. Existen valores inferiores y superiores, según la herencia cultural de cada sociedad, y a ellos se adhiere cada integrante de la comunidad.

\section{LOS VALORES INTERNACIONALES}

Así, podemos hablar de una serie de valores aceptados en el ámbito internacional, y determinados por la ONU y otros organismos internacionales como los grandes valores internacionales del presente, enmarcados dentro de la teoría denominada "Desarrollo sostenible", y estos son:

- Democracia y valores ligados a ella.

- Participación de la sociedad civil.

- Derechos humanos de sujetos sociales: minorías étnicas, mujeres, jóvenes, homosexuales, colonos, etc.

- Culturas indígenas y su problemática.

- Retos a superar: terrorismo, narcopoder, alcoholismo, prostitución, violación, acoso sexual, xenofobia, racismo neofascismo y fundamentalismo.

- Revalorización de la educación y vinculación con la producción empresarial.

Estos son resultado de las tendencias internacionales del comportamiento actual del ser humano y sus grupos sociales. Dentro de esas tendencias destacan los siguientes procesos:

$\checkmark$ El proceso acelerado de urbanización.

$\checkmark$ Desaparición de los espacios rurales.

$\checkmark$ Desplazamiento hacia el sector terciario.

$\checkmark$ Modificación de la familia nuclear.

$\checkmark$ Integración de la mujer al mundo productivo y su emancipación paulatina.

$\checkmark$ Empleo de la tecnología y la electrónica.

$\checkmark$ La educación privada o privatizada.

Tanto las tendencias internacionales (neoliberalismo económico y globalización, entre otras), unido a la propia herencia cultural de cada grupo social, modifican a nivel local el orden e importancia de los valores mencionados, por ello La UNESCO realizo en la década de los noventa un estudio denominado "Los Valores y el Cambio Social", donde se obtuvieron resultados distintos en cada continente, que reflejaba claramente lo antes expuesto, que los valores no tienen la misma importancia o jerarquía en todo el mundo, sino que en cada región se modifican de acuerdo a los individuos que en ella habitan.

Por ejemplo en Europa, donde la visión trabajo en comunidad es poco utilizada desde siempre, están apareciendo nuevos parámetros de valor, con elementos tales como: competitividad. 


\section{LA APLICACIÓN DE LOS VALORES A DIFERENTES SECTORES PRODUCTIVOS}

Vamos a contextualizar la ética en el sector de la construcción, en la ética de los ciudadanos o ética cívica que es la ética de la sociedad civil, entendiendo por sociedad civil "la dimensión de la sociedad no sometida directamente a la coacción estatal" (Cortina, 1998). Esta ética de los ciudadanos, está formada por un núcleo de valores que constituyen la base sobre la que se sustenta la convivencia pacífica de los ciudadanos en sociedades pluralistas.

La ética cívica es hoy un hecho en las democracias occidentales, hemos aceptado ya determinados valores, derechos y actitudes que constituyen unos mínimos compartidos. La ética cívica contiene, además de los valores-guía de cada una de las generaciones de derechos humanos -libertad, igualdad, solidaridad- otros valores importantes, como el respeto activo, el diálogo, la responsabilidad y la justicia (Cerezo, 2005).

La ética cívica es una ética de mínimos referida a lo justo, a lo exigible para una convivencia pacífica, frente a las diversas éticas de máximos, referidas a los diversos ideales de vida buena.

Todo lo dicho en párrafos anteriores, nos lleva a describir los valores fundamentales de la sociedad civil como valores esenciales para la formación en diferentes sectores productivos:

Libertad $\rightarrow$ La libertad es el primer valor a tener en cuenta porque es el presupuesto para la existencia de todos los demás. La libertad es, pues, la condición de posibilidad del sentido de cualquier enunciado que se refiera a lo que debemos hacer.

Igualdad $\rightarrow$ Tiene distintas acepciones: igualdad de todos los ciudadanos ante la ley, igualdad de oportunidades, e igualdad en ciertas prestaciones sociales. La igualdad exige proteger el derecho a la educación, a la atención sanitaria, al trabajo, a la vivienda, a la jubilación, etc. El racismo y la xenofobia son obstáculos ante la conciencia de la igualdad.

Solidaridad $\rightarrow$ La verdadera solidaridad toma en consideración a todos los afectados por la acción de una persona o de un grupo, y solicita que actuemos por una idea de justicia. Pues bien, la solidaridad tiene que ver con el esfuerzo por Ilevar la libertad, la igualdad y el resto de valores morales a aquellos que no pueden disfrutar de esos valores.

Respeto activo $\rightarrow$ En las democracias liberales se entiende que uno de los valores sin los que no es posible la convivencia es la tolerancia. El respeto activo es el interés por comprender a los otros y por ayudar a llevar adelante sus planes de vida.

Diálogo $\rightarrow$ Las soluciones dialogadas a los conflictos son las verdaderamente constructivas, siempre que los diálogos reúnan una serie de requisitos señalados por la ética discursiva. Esto es:

a. convencimiento de que el interlocutor puede aportar algo, por eso está dispuesto a escucharlo.

b. disposición a modificar su posición si le convencen los argumentos del interlocutor. 
c. preocupación por buscar una solución correcta y, por tanto, por entenderse con el interlocutor. (Entenderse no significa conseguir un acuerdo total, pero sí descubrir todo aquello que ya tenemos en común).

d. la decisión final ha de atender intereses universalizables, es decir, los de todos los afectados.

Responsabilidad $\rightarrow$ La responsabilidad hace referencia al hecho de que se le pidan cuentas a una persona por las consecuencias negativas de algo que ha realizado o dejado de realizar, o se le reconozcan las consecuencias positivas. La responsabilidad, como valor ético, tiene que ver con las consecuencias justas o injustas.

Justicia $\rightarrow$ Las tradiciones liberal y social que confluyen en la Declaración Universal de los Derechos Humanos acabarán reconociendo que la justicia consiste en "dar a cada uno las condiciones para vivir en libertad y en igualdad".

De los valores generales que hemos comentado, podemos destacar algunos que surgen como importantes para el ámbito de la formación y los diferentes sectores productivos:

Honestidad $\rightarrow$ se trata de hacer un uso responsable de los recursos y de la información.

Creatividad $\rightarrow$ se trata de aportar constantemente ideas que contribuyan a la mejora y la innovación.

Flexibilidad $\rightarrow$ se trata de asumir como ideas o sugerencias propias las de los demás, reconociendo el cambio como una oportunidad de mejora.

\section{VALORES Y FORMACIÓN EN LA SOCIEDAD DEL CONOCIMIENTO}

Por tanto, el sistema educativo tiene ante sí una exigencia que va mucho más allá de mejorar la calidad una vez que se ha generalizado a los diferentes niveles de enseñanza. Porque se trata de una meta mucho más ambiciosa y estratégica para cualquier país: hilvanar el hilo conductor que necesariamente debe unir la educación, la innovación y el empleo. Este es el gran reto que la sociedad del conocimiento plantea de una forma cada vez más perentoria.

La sociedad y el mundo del trabajo precisan de personas con características acordes con los tiempos, por lo que son muy importantes la capacidad de comunicación, de resolución de problemas y de iniciativa, la capacidad para el trabajo en equipo y el aprendizaje autónomo, las capacidades organizativas y de comprensión del mundo del trabajo y la capacidad de adaptación a los cambios.

Estos nuevos valores ponen de manifiesto la necesidad de introducir cambios de cara a mejorar las inversiones en capital humano. Las compañías comienzan a alejarse del punto de vista estructural para acercarse al cultural y se está impulsando cada vez más el desarrollo de programas que motiven las actitudes de los trabajadores en detrimento de los procesos de trabajo, en la medida en que también el lado humano de las finanzas reporta beneficios.

Aunque muy lentamente, el debate en el ámbito educativo y de la formación comienza a adaptarse a estas nuevas necesidades productivas. Ya en 1993 el Libro Blanco de la Comisión Europea: Crecimiento, competitividad y empleo. Retos y pis- 
tas para entrar en el siglo XXI, señalaba la necesidad de añadir al concepto de saber, como elemento tradicional de la formación, el saber hacer como adquisición a largo plazo de determinadas capacidades y habilidades sociales, entre las que destacaba: "la aptitud para aprender, para comunicar, para trabajar en grupo, para evaluar la propia situación, para formular diagnósticos y hacer propuestas de mejora a todos los niveles, exigiendo autonomía, independencia de espíritu y capacidad de análisis basada en el saber" (Boissonat, 1995: 47).

A pesar de que en el momento presente nos encontramos en un mundo en transición donde conviven dos modelos económicos en proceso de sucesión, si se pueden atisbar las bases en que se enmarcará la formación futura de quienes quieran quedar integrados en el sistema de empleo. Algunos parámetros educativos sobre los que será necesario debatir y lograr acuerdos sociales y administrativos en un futuro próximo van a suponer la necesidad de entrelazar la cultura general y la formación para el empleo. Cada vez se reconoce más la importancia de los conocimientos generales necesarios para dominar los conocimientos profesionales. La cultura general es necesaria para comprender un mundo complejo y para adaptarse a la evolución de la economía y el empleo; esa cultura general inicial es la que incentiva la necesidad en el individuo de formación continua (Cachón y Montalvo, 1999).

Asimismo es importante potenciar la formación polivalente (que posibilite el ejercicio de varias ocupaciones) adquirida a lo largo de la vida activa del trabajador. A través de la misma, deberá adquirir: conocimientos fundamentales, conocimientos técnicos y aptitudes sociales. La formación continua y los aprendizajes extraescolares como lugares fundamentales para la adquisición de dichos saberes y aptitudes deben permitir, además de su aprendizaje, su reconocimiento ágil como competencias válidamente adquiridas (Delors, 1996: 34).

Finalmente, debe aceptarse como necesaria en este mundo permanentemente cambiante, además de los valores mencionados, una serie de destrezas y capacidades que necesitan ser desarrolladas en los trabajadores, de entre las cuales resaltamos: la comunicación verbal y escrita, la capacidad para las relaciones interpersonales, el conocimiento de los procesos de solución de problemas, la destreza en el manejo de herramientas de uso corriente, incluido el manejo de la información, el aprender a valorar la información como elemento básico para la toma de decisiones, la capacidad para mantener un nivel adecuado de autoestima y la capacidad de autonomía personal (Castillo, 1999: 56).

\section{CONCLUSIONES}

Relegar los valores éticos, las capacidades y habilidades sociales y puede decirse lo mismo de todos los valores espirituales y culturales, a un ámbito "especial" fuera de la vida ordinaria, y esperar de los procesos de escolarización su aprendizaje, es algo que desvirtúa la propia adquisición de los mismos. Los valores surgen por la finalidad que tiene toda cultura de proporcionar al ser humano un instrumental simbólico y unas prácticas adecuadas para sobrevivir y desarrollar una vida de calidad. Debería ser normal ver que la asimilación de esos valores se tiene que dar allí donde se generan, dentro de la construcción de la cultura en si misma, en las prácticas 
sociales, económicas y políticas, de producción y reproducción de la vida. En otras palabras, en la vida cotidiana del mundo laboral y de relaciones sociales.

La formación sólo puede venir a continuación, como ayuda para identificar los contenidos éticos y espirituales en esas prácticas de la vida diaria y del desempeño profesional.

En la medida en que seamos capaces de resolver e integrar los indicadores que hemos mencionado (valores, habilidades sociales y capacidades) estaremos trascendiendo la mera formación técnica y teórica y estaremos iniciando una formación más sólida y acorde con el futuro que nos espera, lo que ayudará a los sujetos, además de a ser trabajadores polivalentes, a ser personas y ciudadanos responsables.

\section{Bibliografía}

BARBADILLO GRIÑÁN, P. (1997). Extranjería, racismo y xenofobia en la España contemporánea: La evolución de los setenta a los noventa. Madrid: Centro de Investigaciones Sociológicas/ Siglo XXI.

BOISSONAT, J. (1995). Le travail dans vingt ans (Rapport de la Commission presidée par...). París: Odile Jacob/La Documentation Française.

BOLADERAS, M. (1993). Libertad y tolerancia. Éticas para sociedades abiertas. Barcelona: Publicaciones de la Universidad de Barcelona.

BONI, A. y FERRERO, G. (1997). Introducción a la Cooperación para el Desarrollo. Valencia: Universidad Politécnica de Valencia.

BUXARRAIS ESTRADA, M. R. et al. (1993). El racismo: el interculturalismo en el currículum. Barcelona: Asociación Maestros Rosa Sensat.

BUXARRAIS, M. R. (1997). La formación del profesorado en educación y valores. Propuesta y materiales. Bilbao: Desclée de Brower.

CACHÓN, L y MONTALVO, Má. D. (1999). Educación y formación a las puertas del siglo XXI. La formación continua en España. Madrid: Editorial Complutense.

CASTILLO, J. J. (1999). El trabajo del futuro. Madrid: Editorial Complutense.

CEREZO, P. (ed.) (2005). Democracia y virtudes cívicas. Madrid: Biblioteca Nueva.

CORTINA, A. (1994). La ética de la sociedad civil. Madrid: Anaya

CORTINA, A. et al. (1998). Educar en la justicia. Valencia: Generalitat Valenciana.

DELORS, J. (1996). La Educación encierra un Tesoro. Informe a la Unesco de la Comisión Internacional sobre la educación para el siglo XXI. Madrid: Santillana.

FABELO, J. R. (1996). La crisis de valores, conocimiento, causas y estrategias de superación. La formación de valores en las nuevas generaciones. La Habana, Cuba: Ed. Revolucionarias.

GIARINI, O y LIEEDTKE, M. (1998). El dilema del empleo. El futuro del trabajo (Informe al Club de Roma). Barcelona: Círculo de Lectores / Galaxia Gutemberg.

HABENIUS, A. (1996). "Making Civil Society Work, Promoting Democratic Development: What Can Sates and Donors Do?" World Development, 24(10), 1621-1996

KOHLBERG, L. (1987). "El enfoque cognitivo-evolutivo de la educación moral", en Jordán, J. A. y Santolaria, F. F. (edit.). La educación moral, hoy. Cuestiones y perspectivas. Barcelona: PPU, pp. 85-114. 
LAZARTE, R. (2005). Max Weber: ciencia y valores. Rosario; San Fe, Argentina: Homo Sapiens Edics.

MÉNDEZ, J. M. (1997). Tabla de valores éticos. Madrid: Estudios de Axiología.

ORACIÓN, M. (coord.). (2005). La construcción de la ciudadanía en el siglo XXI. Barcelona: Octaedro.

ORTEGA, P., MÍNGUEZ, R. y GIL, R. (1994). Educación para la convivencia: la tolerancia en la escuela. Valencia: Nau Llibres.

PASCULA, A. V. (1995). Clarificación de valores y desarrollo humano. Madrid: Narcea.

PAYÁ, M. (1997). Educación en valores para una sociedad abierta y plural. Bilbao: Desclée de Broker.

PUIG, J. M. y MARTÍNEZ, M. (1989). Educación moral y democracia. Barcelona: Alertes.

SUÁREZ PERTIERRA, G. y CONTRERAS MAZARÍO, J. M. (2005). Interculturalidad y educación en Europa. Valencia: Tirant lo Blanch. 